\title{
LA CONVENTION COLLECTIVE DE TRAVAIL EN DROIT TURC ET EN DROIT SUISSE
}

\author{
Par Alexandre BERENSTEIN \\ Professeur à la Faculté de droit \\ de l'Université de Genève
}

\section{INTRODUCTION}

Le Code des obligations turc contient, au sujet de la convention collective de travail, deux articles, les articles 316 et 317 , qui sont la traduction des articles 321 et 322 du Code suisse des obligations, dans sa teneur de 1911 (1). Mais ces dispositions coexistent aver celles qui résultent de la loi spéciale No 275 du 15 juillet 1963 sur les conventions collectives de travail (2), modifiée le 23 juillet 1964. Le droit turc connaît ainsi dsux systèmes différents de contrats collectifs ou conventions collectives de travail, basés l'un sur le code, l'autre sur la loi spéciale.

La première observation qui s'impose lorsque i'on compare l'institution de la convention collective en Suisse avac cette institution en Turquie est la suivante:

En Suisse, les conventions collectives sont nées de la pratique. C'est après que les contrats collectifs eurent pris un certain développement que le législateur a entendu les réglementer dans le coda de 1911. Le besoin de cette législation était très vivement ressenti. La loi fédérale a d’ailleurs été précédée de lois cantonales. notamment des lois genevoises de 1900 et de 1904. Par la suite,

(1) La présente étude est basée essentiellement sur le rapport du professeur Turhan ESENER, présenté à la semaine juridique turco-suisse d'Ankara. Nous avons consulté aussi. le rapport du professeur $F$. $H$. SAYMEN in Actes du 2 ème Congrès international de droit du travail (Genève 1957) et le cours du professeur Kemal ELBIR donné en 1965 à la Faculté internationale pour l'enseignement diu droit comparé.

(2) B. I. T., Série législative - 1963 Tur. 2. 
l'institution a continué de se développer; elle a pris des formes nouvelles qui ont apporté des solutions jurisprudentielles. D'autre part, son évolution s'est heurtée à certaines difficultés juridiques, et il a paru opportun de reviser les dispositions légales pour tenir compte de cette situation et pour répondre aux nécessités nées le la pratique. Quant à l'extension des contrats collectifs aux tiers, elle avait été prévue dès 1919 dans la loi fédérale portant régle. mentation des conditions du travail, mais cette loi, adoptée par l'Assemblée fédérale, a été rejetée par le peuple en 1920, et il a fallu attendre la dernière guerre, soit l'année 1941, pour qu'un nouveau texte sur cet objet pût être adopté par l'Assemblée fédérale et entrer en vigueur (arrêté fédéral du ler octobre 1941 permettant de donner force obligatoire générale aux contrats co'lectifs de travail). L'arrêté de 1941, modifié en 1943, a été remplacé ensuite par la loi fédérale. du 28. septembre 1956 permettant d'étendre aux tiers la convention colisctive de travail. (C'est cetre même loi qui a abrogé les articles 322 et 323 du Code des obligations et les a remplacés par sept nouveaux articles).

En Turquie, en revanche, il semble que l'on n'ait pas connu de contrat collectif avant l'adoption du Code des obligations, et, malgré les dispositions de ce code, les rares contrats collectifs qui avaient été ébauchés par certains syndicats n'étaient «que deis manifestations sporadiques toujours sans envergure et souvent sans lendemain» (1). C'est précisément en vue de développer et d'encourager l'institution du contrat collectif que le législateur ture a, après l'adoption en 1.961 de dispositions constitutionnelles traitant de ce problème, édicté la législation nouvelle. Et le développement des conventions collectives semble être une conséquence de la législation, plus qu'une conséquence de l'évolution spontanée.

Si l'on examine la loi de 1963, qui ne traite pas seulement de la convention collective de travail, mais aussi des conflits collectifs, on constate qu'elle s'inspire, dans ses dispositions sur les convertions collectives, à la fois des législations suisse, française, allemande et américaine, mais qu'elle comporte différentes caractéristiques originales. Elle permet de conclure dans des conditions données des conventions collectives de travail qui emportent

(1) Saymen, loc. cit. p. 282. 
tains effets déterminés - tandis que les contrats collectifs linaires, qui sont conclus sur la base du C.O. emportent des ets différents.

\section{L'OBJET DE LA CONVENTION}

Quant à l'objet de la convention, la loi turque de 1963 s'inspire s nettement del l'art. 322 revisé du C.O. suisse, mais sous réserve $\mathrm{n}$ point important. La loi suisse de 1956 a élargi la notion de sonvention collective. Auparavant, en vertu de l'art. 322, étaient ls considérés comme «contrats collectifs de travail» les contrats blissant «des règles relatives aux conditions du travail», c'est-à₹ des contrats réglementant le contenu des contrats individuels travail. Sous l'empire de la loi de 1956, il suffit que la convention tienne des dispositions sur les rapports entre employeurs $e_{:}$ vailleurs pour qu'elle puisse être une «convention collective de vail».

A vrai dire, l'article 322 nouveau du C.O. suisse est mal igé, et la loi turque, en le reprenant en grande partie, ne souffre ; de la même erreur de rédaction. En effet, lorsque l'article 322 te (alinéa 1) que la convention collective est un contrat par uel sont établies des clauses sur la conclusion, le contenu et rtinction des contrats individuels de travail, il semble donner ? définition, mais il s'agit d'une fausse définition - puisque, par suite, dans l'alinéa 2 du même article, il est dit que la convention lective peut ne pas contenir de telles clauses, pourvu qu'elle ıtienne des dispositions quelconques sur les rapports entre ployeurs et travailleurs. En revanche, la loi turque, en ne repreit pas la phrase de l'alinéa 2 in fine de l'art. 322 revisé du C.o. sse : «Elle peut même se limiter à ces autres dispositions», dmet pas comme étant des conventions collectives les textes . ne contiennent pas de clauses relatives à la conclusion, au Itenu et à l'extinction des contrats de travail individuels. C'est si qu'une convention qui se borne à régler les rapports entre ;anisations de travailleurs et organisations d'employeurs consde une convention collective en Suisse; elle ne l'est pas en rquie. 


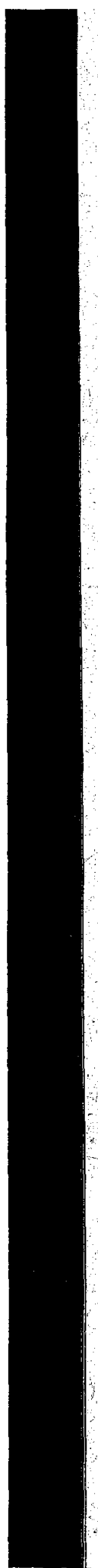

\section{LES PARTIES A LA CONVENTION}

Quant aux parties, l'article ler de la loi turque reprend à peu près les mêmes termes que l'article 322 du C.O. suisse - qui, sur ce point, n'est d'ailleurs pas différent de la plupart des autres législations nationales - pour désigner les parties à la convention collective: la convention est conclue «entre des organisations de travailleurs et des organisations d'employeurs ou des employeurs s. Mais il ajoute que ces organisations ou ces employeurs individuels doivent être ceux qui sont visés à l'art. 7 de la même loi. Or. cette dernière disposition restreint le droit de conclure une convention collective au sens de la loi aux fédérations ou syndicats de travailleurs représentant la majorité des travailleurs occupés dans une branche d'activité ou dans un ou plusieurs établissements, et d'autre part aux fédérations ou syndicats d'employeurs dont les mẹmbres occupent la majorité (1) des travailleurs occupés dans la branche d'activité. Cependant, un syndicat d'employeurs peut conclure une convention pour les établissements appartenant ${ }^{\circ}$ à ses membres, de même qu'un employeur individidel peut le faire pour son propre établissement. Sous réserve de cette dernières disposition, qui facilite la conclusion de conventions collectives aux employeurs, intervient ici le critère de la représentativé, que re connaît pas le droit suisse. Certes, pour que l'extension du champ d'application d'une convention collective puisse être requise en droit suisse, il faut notamment que les travailleurs liés par it convention forment en principe la majorité des travailleurs auxquel.s la convention dioit s'appliquer. Mais la loi suisse ne contient aucune disposition limitant en quoi que ce soit le droit de négocier une convention collective à tel syndicat déterminé. La libsrté la plus absolue est de règle dans ce domaine. C'est seulement une fois la convention négociée et conclue que la question des majorités 5 pose, si l'on désire demander l'extension du champ d'application.

Le législateur suisse s'est donc efforcé de limiter le moins passible la liberté des parties. Les seules mesures prises ont pour'

(1) Nous nous référons sur ce point au rapport du prof. Esener, la traduction française de l'art. 7 de la loi No 295 du 15.7.1963 publiée dans 1a «Série législative» n'indiquant pas la condition de majorité en ce quí concerne le syndicat de travailleurs constitué pour une branche d'activitê. 
but de protéger les minorités syndicales et les dissidents. Le droit turc intervient en sens inverse.

Sur ce point, la loi turque présente quelque analogie avec la Iégislation française, selon laquelle les conventions collectives n€ peuvent faire l'objet d'une décision d'extension que si elles ont été négociées selon une procédure spéciale. Cependant, en France, la négociation, dans ce cas, ne se fait pas avec unè seule organisation, qui serait considérée comme la plus représentative. Ces conventions collectives sont négociées au sein d'une commission comprenant des représentants de toutes les organisations professionnelles considérées comme étant le plus représentatives (article 31 f, livre ler, Code du travail).

Mais le système turc semble surtout s'être inspiré de celui des Etats - Unis d'Amérique, où la loi Wagner de 1935 sur les relations du travail (Labor Relations Act) a prévu que dans chaque bargaining unit, seul le syndicat le plus représentatif serait autorisé à conclure une convention collective. Aux Etats - Unis, cependant, les litiges qui ont pour objet la détermination du syndicat le plus représentatif sont tranchés par un vote auquel il est procédé sotis le contrôle de l'administration. En Turquie, c'est une décision administrative (selon les cas, direction régionale du travail ou Ministère du travail) qui tranche les conflits de ce genre, sous réserve d'un recours judiciaire. Mais il convient d'observer que la décision, soit administrative, soit judiciaire, doit être prise sur le plan de l'établissement dans les trois jours, sur le plan d'une branche d'activité dans les six jours dès le dépôt de la demandre ou du recours. Certes, la brièveté du délai comme telle ne peut qu'être bénéfique aux intéressés. Mais l'on peut se demander si, Jans le délai de trois ou de six jours prévu par la loi, il est possible à l'administration ou à la juridiction saisie de se prononcer m toute connaissance de cause sur l'importance respective des rganisations en présence.

Il ne nous appartient pas d'émettre un jugement de valeur sur l'application de l'un ou l'autre des systèmes, du système de la liberté de contracter existant en Suisse et du système de la limitation du droit de contracter à l'organisation la plus reprèsentative, tel qu'il se présente en Turquie. 
En effet, le choix entre les deux systèmes ne peut procéder d'une vue théorique de l'esprit, mais des conditions sociales, qui sont toutes différentes de part et d'autre, et l'on ne peut donc comparer des situations qui ne sont pas comparables. Tout ce que l'on peut examiner si l'on veut demeurer dans le cadre de l'analyse juridique, c'est la question de savoir si l'un et l'autre de ce.s systèmes sont logiquement conçus et s'ils répondent aux exigences qui leur sont posées. Sous cet angle, on peut se demander si les litiges relatifs à la représentativité des organisations profession. neles peuvent être jugés en Turquie compte tenu de tous les facteurs qu'il y a lieu de considérer en l'occurrence.

De toute façon, il est clair que la législation turque attache une très grande importance à la capacité de contracter. C'est ainsi que cette capacité est refusée par la jurisprudence - nous apprend le professeur ESENER - aux confédérations syndicales, contraire. ment à ce que prévoit par exemple la loi allemande, qui l'accorde en principe aux Spritzenorganisationen $(\S 2$ al. 3 dè la loi du 9 avril 1949).

En Suisse, tout cela est en dehors de la loi: Le législateur n'a pas voulu réglementer les négociations collectives. Il s'est contenté de réglementer leur résultat: les conventions collectives. Toute liberté est laissée aux organisations de négocier avec qui elles entendent et avec qui elles peuvent négocier. "Les mesures prises dans le cadre de la liberté de contracter sont admissibles: même si pour le surplus elles sont utilisées comme arme dans la lutte que se livrent entre elles les différentes associations ouvrières pour conquérir la prédominance», a déclaré le Tribunal fédéral dan:s son arrêt Muller du 13 septembre 1949 (1). La liberté dans ce domaine ne trouve, d'après le Tribunal fédéral, qu'une limite : «L'employeur ou l'association patronale qui refuseraient, sans aucun motif raisonnable, d'entrer en discussion avec une association ouvrière en vue de la conclusion d'un contrat collectif danis l'intention évidente d'affaiblir la situation des ouvriers et de se procurer, de cette façon, un avantage pour eux - mêmes, commettraient un acte illicite et contraire aux moeurs, parce que leur attitude aurait pour effet de priver les ouvriers de la protection

(1) A T F 75 II 327, Journal des Tribunaux 1950 I 181. 
voulue par la loi et irait à l'encontre d'une des idées fondamentales de la législation sur le contrat collectif de travail» (arrêt F.O.T.F. du 25 mai 1947) (1). Cependant, cette affirmation de principe du Tribunal fédéral n'a jusqu'ici jamais trouvé d'application dans la pratique. Si l'employeur ou la fédération d'employeurs se refusent à négocier, le syndicat de travailleurs, en dehors du recours à des moyens de pression tels que la grève, ne peut que faire convoquer la partie patronale devant l'office cantonal de conciliation - tout au moins lorsqu'il s'agit d'un conflit touchant des ouvriers d'entreprises industrielles-, conformément à l'art. 31 de la loi sur lp travail dans les fabriques, ou, le cas échéant, demander la constitution d'un office fédéral de conciliation (art. ler de la loi fédérals du 12 février 1949) afin de tenter d'amener les employeurs à acospter de négocier.

Il convient de relever en passant que, si la loi turque régiemente très strictement les conflits du travail, il n'en est pas de même en Suisse, où les dispositions relatives à la constitution des offices de conciliation sont à peu près les seules en la matière. I1 n'existe pas non plus, en Suisse, de dispositions sur ia constitution les syndicats, qui sont des associations ou des sociétés coopérativesi régies par le droit commun, alors qu'en Turquie les syndicats sont égis par une loi spéciale, la loi No 274 du 15 juillet 1963 (2).

\section{LES EFFETS JURIDIQUES DE LA CONVENTION}

\section{1 - En général :}

Les effets juridiques varient en Turquie selon que l'on a ffaire à un contrat collectif régi par le C.O. ou à une convention ollective régie par la loi No 275. Certes, les effets généraux de as catégories de textes sur les rapports entre les parties aux conats individuels de travail ne diffèrent pas essentiellęment. La invention collective de travaii emporte, en outre, d'autres effets, stamment l'autorisation de décréter une grève ou un lock - out en s de violation de la convention par le cocontractant. Il est rappeló ce propos que le problème ne se pose pas sous le même angie

) A T F 74 II 163, Journal des Tribunaux 1949 I 307.

) B.I.T. Série législative - 1963 - Tur. 1. 
en Suisse, puisque, au regard du droit public, grève et lock-out $\mathrm{y}$ sont en principe licites.

Mais à cela s'ajoute le fait que la «convention collective de travail» du droit turc a une valeur supérieure à celle du «contrat collectif» du Code — dit «contrat général»--. sauf les dispositions de ce dernier qui seraient plus favorables aux travailleurs (art. $3 \S 2$ ).

Tout comme en Suisse, en Turquie les effets juridiques de la convention collective en ce qui concerne les rapports individuels de travail ne se manifestent qu'entre personnes liées de part e! d'autra par la convention collective: c'est-à-dire entre un travailleur membre du syndicat contractant et un employeur membre de l'organisation patronale contractante - ou un travailleur membre et un employeur signataire de la convention à titre individuel. En revanche, la convention n'affectera pas, en principe, les rapports entre l'employeur lié par la convention et le travailleur non membre du syndicat contractant.

On peut remarquer d'emblée à ce sujet qu'il semble qu'il y ait, en Turquie, une certaine discordance entre les règles sur la capacité de contracter et la conception des effets juridiques de la convention. Généralement, lorsque l'on exige que, pour pouvoir contracter, un syndicat représente la majorité des travailleurs intéressés, on le fait parce que l'on entend qu'il puisse contracter pour l'ensemble des travailleurs et, par conséquent, également pour la minorité qui n'y est pas affiliée. C'est, notamment, ce que prévoit aux Etats - Unis la loi Wagner (art. 9 a (1).

Il apparaît à première vue difficile de justifier, d'une part, le monopole accordé à l'organisation la plus représentative en matière de négociation et, d'autre part, le fait que cette dernière ne peut négocier que pour ses membres, les minoritaires étant exclus de toute protection résultant de la convention collective. Mais il est vrai que les syndicats minoritaires peuvent négocier des «contrats généraux», fondés sur le C.O. et qui ne sont pas soumis aux mêmes limitations.

(1) Cf. Generald A. Brown, in Labour Relations and the Law. 'British Institute Studies in International and Comparative Law, No 2, p. 54. 
Il importe de relever aussi que les effets juridiques de la convention collective de travail peuvent, en Turquie, être étendus aux tiers par l'emploi de daux procédés: le paiement d'une contribution de solidarité et l' extension par décision des pouvoirs publics.

\section{2 - La contribution de solidarité :}

Comme la convention collective elle-même, l'institution dite de la «contribution de solidarité» est née de la pratique en Suisse, tandis qu'elle a été introduite en Turquie par le législateur. Mais la conception qui est à la base de cette contribution n'est pas la mêms dans l'un et l'autre de ces pays.

En Suisse, la contribution de solidarité est un élément qui accompagne quelquefois -mais qui n'accompagne pas nécessairement- une autre institution : celle de la «soumission» ou de la «participation» à la convention. C'est aussi de la pratique qu'esi. sortie l'institution de la «soumission». Les parties au contrat collectif faisaient signer aux outsiders une déclaration de soumission; jar laquelle l'employeur ou le travailleur dissident s'engageaient $i$ se «soumattre» au contrat collectif et $\dot{a}$ en observer les clauses. Jeux-ci étaient, dès lors, considérés comme pouvant bénéficier des lauses du contrat. La doctrine admettait, cependant, que cette léclaration de soumission n'entraînait pas d'effet normatif, en ce iens que l'intéressé n'aurait pu se prévaloir, dans un litige avec on cocontractant, des termes de la convention collective. L'instiution de la soumission a été généralement liée à celle de la clans; le fidélité, ou d'exclusivité conventionnelle, l'employeur lié par la onvention s'engageant à ne pas occuper des travailleurs qui ne ont pas membres de l'organisation syndicale contractante ou n'ont as souscrit une déclaration de soumission; quant au travailleur é par la convention, il s'engageait à ne pas travailler chez un mployeur non membre de l'organisation patronale contractanic u n'ayant pas souscrit une déclaration de soumission. La loi de 356 a remplacé la «soumission» par la «participation», qui ses fférencie de celle-là en ce que, prévue expressément par la loi, le emporte un effet normatif. Les effets normatifs de la conintion sont, en effet, étendus aux «participants», dont la déciaเtion doit être remise par écrit et doit recevoir l'accord écrit des sux parties (art. 322 bis et 322 ter C.O.). Une contribution de 
solidarité est quelquefois exigée de l'employeur ou du travailleur participant, auxquels on demande de verser cette somme en contrepartie des sacrifices effectués par les membres des organisations professionelles qui, par leurs cotisations, ont permis l'obtention, pour les membres de la profession, d'avantages substantiels (1).

En Turquie, l'institution de la contribution de solidarité ne concerne que les travailleurs, et non les employeurs. Sa nature juridique est aussi toute différente. Elle n'est pas liée à une déclaration de soumission, ou de participation, mais le paiement de la contribution emporte automatiquement l'application de la convention au «contribuable». Et surtout, il n'y a, en Turquie, pas de différence essentielle entre la contribution de solidarité et la cotisation syndicale. C'est d'autant plus vrai que la jurisprudence de la Cour de cassation a décidé, par un arrêt du 3 décembre 1964, que les non syndiqués qui entendent bénéficier d'une convention et payer la contribution de solidarité doivent obtenir l'approbation du syndicat. contractant. Il s'agit donc d'une convention passée entre le syndicat et son membre, ol, si on le préfère, d'une adhésion au syndicat en qualité de membre de deuxième zone.

En revanche, en Suisse, la participation consiste avant tout dans une liaison entre le participant et le système conventionnel, et non avec le syndicat. La loi précise d'ailleurs que les contributions ne: peuvent être utilisées au profit de l'une seulement des parties à la convention collective (art. 322 bis, al. 2, C.O.).

On ne voit donc pas très bien l'utilité de l'institution de la contribution de solidarité en droit turc, si ce n'est que l'intéressé peut ainsi payer une contribution inférieure à la cotisation syndicale en bénéfiçiant des avantages de la convention: la loi turque limite aux deux tiers de la cotisation syndicale le montant admis. sible de la contribution (art. $7 \& 3$ loi No 275). Il est rappelé que, de son côté, le droit suisse laisse au juge la faculté de «ramener à de justes limites» les contributions excessives.

\section{3 - L'extension par décision des pouvoirs publics :}

Si l'institution de la «contribution de solidarité» permet d'étendre, par dès moyens de droit privé, le champ d'application

(1) Voir à ce sujet notre étude in Revue internationale du travail, février 1962, p. 111. 
de la convention collective, le droit turc, comme le droit suisse, connaît aussi le système de l' extension aux tiers par décision des pouvoirs publics. On notera que la loi turque ne prévoit pas, comme le fait la loi suisse, qu'une telle extension ne peut être décidée que sur requête de toutes les parties contractantes (art. ler de la loi du 28 septembre 1956); d'autre part il suffit, pour qu'elle puisse être décrétée, que les travailleurs auxquels s'applique la convention constituent la majorité des travailleurs de la branche d'activité -alors qu'én Suisse il faut, en outre, que soit réunie la majorité des employeurs et que les employeurs liés occupent eux - mêmes la majorité de tous les travailleurs (art. 2 chiffre 3 ). De plus, la loi suisse est moins rigide du côté des travailleurs, puisqu'elle permet qu'il soit dérogé à la règle de la majorité des travailleurs - dérogation qui, bien qu'étant «exceptionnelle» aux termes de la loi, est de pratique courante- ce qui s'explique facilement du fait lu grand nombre de travailleurs étrangers non syndiquées qu' zccueille actuellement la Suisse.

\section{4 - La sortie d'un membre de l'association contractante :}

Il convient de relever aussi, en ce qui concerne l'effet des lauses normatives de la convention collective, que la loi turque dmet expressément, ce que ne fait pas la loi suisse, que lorsqu'un ravailleur ou un employeur membre d'une organisation contracante quitte son organisation, il demeure lié par la convention. 'employeur, dans le cas d'une convention conclue pour une durée ıdéterminée, peut toutefois résilier la convention à l'expiration 'une année à compter de la date de sa sortie de l'organisation, t moyennant l'observation d'un préavis de trois mois (article 6 de $\checkmark$ loi No 275).

Sous réserve de cette dernière exception, le principe résultant a l'article 6 de la loi turque concorde avec la situation juridique zistant en France (art. 31e, alin. 1, livre 1er du Code du travail) L) et en Allemagne ( $\$ 3$, loi du 9 avril 1949) (2), où le membre rrtant demeure lié par la convention. En revanche, en Suisse, spinion dominante admet que le membre sortant d'une organi. :tion contractante n'est, en principe, plus lié par la convention

) Cf. Brun et Galland, Droit du travail, p. 740 No III - 74, et Despax, Convenitons collectives, p. 280 , No 176 .

) Cf. Hueck - Nipperdey - Tophoven, 4 ème édit., ad $\&$ 3, No 14, p. 190. 
collective, mais cette opinion semble être fondée surtout sur l'ancienne doctrine allemande (1). La loi turque paraît, sur ce point, plus conforme à la nature juridique de la convention collective, qui devrait s'imposer aux membres des associations sans qu'ils puissent s'y soustraire en se retirant de l'organisation à laquelle ils appartenaient lors de la conclusion de la convention ou ultérieurement.

\section{5 - La «survie» de la convention}

Enfin, la loi turque prévoit aussi l'effet prolongé ou la «survì» de la convention collective en ce sens que les normes qui y sont contenues continuent après son extinction à porter effet sous ia forme d'un contrat individuel de travail (art. 3 \$2), ce qui est également conforme à la loi allemande ( $\$ 4$ al. 5), alors que la $1 \mathrm{i}$ suisse ne règle pas cette question et que l'opinion dominante se prononce en sens inverse (2).

\section{LES CONFLITS DE CONVENTIONS COLLECTIVES}

Le professeur ESENER, dans son remarquable rapport, a exposé en détail les problèmes créés en Turquie par les conflits de conventions collectives de travail.

En Suisse, le pluralisme syndical pose également, à cet égard, cartains problèmes, mais ils sont généralement assez facilement. résolus.

Dans divers cas, l'organisation d'employeurs négocie avec 'e syndicat le plus important et, avec l'autorisation de ce syndicat, conclut ensuite une convention dite "parallèle» avec le syndicat minoritaire, convention qui contient les mêmes règles normatives que la convention principale. Ou encore les différents syndicats s'associent pour la discussion avec l'organisation patronale et signent en commun la convention. Ou bien, enfin, les organisations minoritaires, une fois achevée la discussion entre l'organisation patronale et le syndicat le plus puissant, sont autorisées à apposer leur signature au-dessous de la convention.

(1) Oser - Schönenberger. Das Obligationenrecht, 2e éd. vol. 2, ad art. 323 No 26, p. 1206; Schweingruber, Kommentar zum Gesamtarbeit3vertraig, ad art. 323 No 4 p. 57.

(2) Oser - Schönenberger, loc. cit. ad, art. 323 No 11, P. 1201 
pluralité de conventions peut aussi résulter en Suisse du 'à une convention nationale, qui est généralement une con «cadre», s'ajoutent des conventions régionales ou locales. on dominantei admet qu'en cas de conflit les dispositions i favorables aux travailleurs l'emportent, par analogie avcc se passe dans le cas de conflit entre la convention collectiv ontrats individuels de travail (art. 323 alinéa 2 C.O.).

is en Turquie, la plupart des problèmes qui se posent à cet ésultent de dispositions spéciales de la loi concernant les ions collectives de travail - et notamment de l'unicité oblide la convention, qui doit être conclue avec le syndicat le présentatif. La loi suisse ne contenant pas de telles dispoil n'y a pas en Suisse de tels problèmes à résoudre.

\section{ONSIDERATIONS FINALES}

conclusion, on peut observer que les lois turque et suisse $t$ sur des conceptions radicalement différentes. En Suisse, isse aux associations la plus grande liberté dans l'élaboraticn ventions collectives, sous réserve de certaines dispositions s à la protection des syndicats minoritaires et des dissiin Turquie, en revanche, la loi contient de nombreuses dis$\mathbf{s}$ de caractère impératif, auxquelles les associations rie déroger. Les négociations collectives elles-mêmes sont ntées par l'Etat.

utre part, la loi turque ne s'occupe que des dispositions invention collective emportant un effet normatif et, notam. e leur incidence juridique sur les contrats individuels de Elle ne traite pas des dispositions de caractère contraciuel mment, de celles qui concerneraient l'organisation des rapatre associations. On sait que la loi suisse accorde, au e, une grande importance à cet aspect du problème, quii 'effleuré dans l'article ler de la loi turque No 275 . Celui-ci à son $\S 2$, quie la convention collective peut contenir d'autres ons concernant les rapports entre employeurs et travailles droits et obligations réciproques des parties - disposition de l'art. 322 , al. 2 et 3 , C.O. suisse. Mais la Ioi turque, zoir mentionné cette possibilité, ne s'étend pas plus avant modalités de l'organisation des rapports entro associations. à cet égard, aussi, une divergence fondamentale entre les z̧islations, divergence qui a, sans doute, son fondement différences existant dans la situation de fait. 